\title{
Effective therapy of epidermal growth factor receptor inhibitor-associated dermatologic side effects in a patient with metastatic colorectal cancer: a case report and review of literature
}

\author{
Alicja Góralczyk¹, Małgorzata Sokołowska-Wojdyło', Anna Kowalczyk², Aneta Szczerkowska-Dobosz¹, \\ Wioletta Barańska-Rybak ${ }^{1}$
}

\author{
${ }^{1}$ Department of Dermatology, Venereology and Allergology, Medical University of Gdansk, Poland \\ Head: Prof. Jadwiga Roszkiewicz MD, PhD \\ 2Department of Oncology and Radiotherapy, Medical University of Gdansk, Poland \\ Head: Prof. Jacek Jassem MD, PhD
}

Postep Derm Alergol 2012; XXIX, 4: 324-329 DOI: $10.5114 /$ pdia.2012.30475

\begin{abstract}
Overexpression and impaired signaling of the epidermal growth factor receptor (EGFR) are involved in the cancerogenesis. The EGFR inhibitors such as cetuximab have shown efficacy in the targeted therapy of neoplasm. The acneiform rash has been revealed as the most common side effect of treatment, usually occurring within 2 weeks after therapy onset. We report perifollicular pustules and papules with impetiginization in a 63-year-old patient with metastatic colorectal cancer treated with cetuximab. The neoplasm was diagnosed in 2008 and treated surgically (resection of the sigmoid). After 3 years, a combined therapy was administered due to the disease progression. It consisted of cetuximab and FOLFOX (Folinic acid, Fluorouracil, Oxaliplatin) chemotherapy regimen. The effective dermatologic management (with systemic doxycycline and topical tacrolimus and fusidic acid) of the skin toxicity allowed for maintenance of oncologic treatment. Despite the proper dermatological therapy, the described side effects may persist, and the exacerbation of skin lesions can cause discontinuation of treatment of cancer with those modern biological agents (EGFR inhibitors and other kinase inhibitors). When discontinuation is not necessary - cutaneous symptoms adversely affect the quality of life. However, there are reports that recognition of the skin lesions in the course of the above therapies is a good prognostic factor, confirming effectiveness of treatment. Moreover, the association between genetic polymorphisms, the risk of treatment adverse effects and the response of the tumor to monoclonal antibody targeted therapy is well established. This leads to the increasing role of pharmacogenetics in the management of neoplasms.
\end{abstract}

Key words: epidermal growth factor receptor inhibitors, cetuximab, dermatologic toxicity, papulopustular rash.

\section{Introduction}

The epidermal growth factor receptor (EGFR) is a tyrosine kinase receptor composed of an extracellular ligand-binding domain, a trans membrane segment and an intracellular protein kinase. The EGFR regulates cell growth and differentiation. Its overexpression is commonly found in neoplastic cells and associated with poor prognosis for the patient. Disruption of EGFR-mediated signaling leads to cancerous cell proliferation, migration, stromal invasion, resistance to apoptosis and angiogenesis $[1,2]$.
As a molecular target for cancer therapy EGFR may be inhibited with anti-EGFR-specific monoclonal antibodies or small molecule tyrosine kinase inhibitors. They are effective in the treatment of advanced, metastatic or recurrent tumors like colorectal carcinoma, non-small cell lung cancer, squamous cell carcinoma of the head and neck, pancreatic cancer and breast cancer [3, 4]. The EGFR inhibitors are used alone or in combination with other drugs or radiation. While agents targeting EGFR lack many of the severe systemic side effects associated with traditional cytotoxic chemotherapy, they induce cutaneous

Address for coresspondence: Alicja Góralczyk, Department of Dermatology, Venereology and Allergology, Medical University of Gdansk, 7 Dębinki, 80-210 Gdansk, Poland, phone: +48 500046 151, e-mail: a.goralczyk@gumed.edu.pl 
reactions. All EGFR inhibitors can cause dermatological toxicity [5]. Other side effects of this antineoplastic therapy include: infusion reactions, diarrhea, hypomagnesaemia, cardiopulmonary arrest [6].

\section{Case report}

A 63-year-old woman with metastatic colorectal cancer was referred to a dermatologist due to severe papulopustular perifollicular rash induced by treatment with cetuximab.

The neoplasm was diagnosed in 2008 and treated surgically by the resection of the sigmoid. In 2011, the elevated blood concentration of carcinoembryonic antigen (CEA) was observed. Single photon emission computed tomography (SPECT) scan revealed a disease progression - metastasis in the liver (measuring $3.5 \mathrm{~cm}$ ), as well as the mediastinal, axillary and mesenteric lymph node metastases. A combined therapy was initiated on 19 September 2011, including cetuximab given intravenously every week at a dosage of $400 \mathrm{mg} / \mathrm{m}^{2}$ for the first administration followed by $250 \mathrm{mg} / \mathrm{m}^{2}$ from the second administration on, and FOLFOX (Folinic acid, Fluorouracil, Oxaliplatin) chemotherapy regimen given intravenously every other week.

Six months after treatment onset the patient sought advice from the dermatologist due to facial skin changes associated with pruritus and tenderness. Physical examination showed diffuse erythema, edema of the eyes and face, as well as the perifollicular pustules and papules with impetiginization. The diagnosed papulopustular rash was classified as grade 2 according to the National Can-

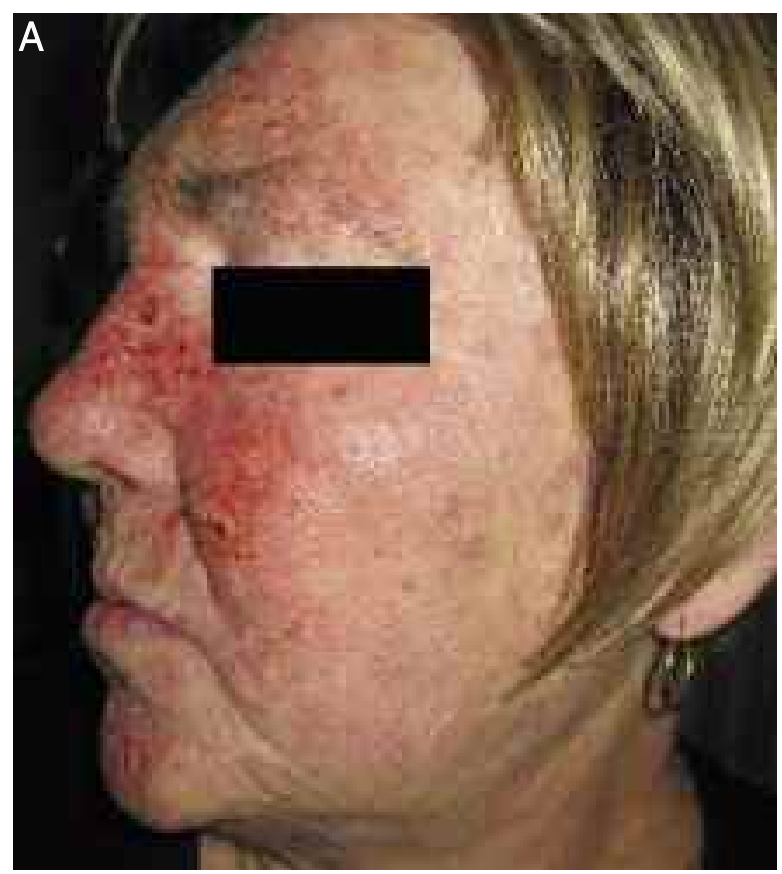

cer Institute's Common Terminology Criteria for Adverse Events version 4.03, and by the use of MASCC (Multinational Association of Supportive Care in Cancer) scale as grade 2 as well [7].

The medical history of the patient revealed that cutaneous rash had developed after the third administration

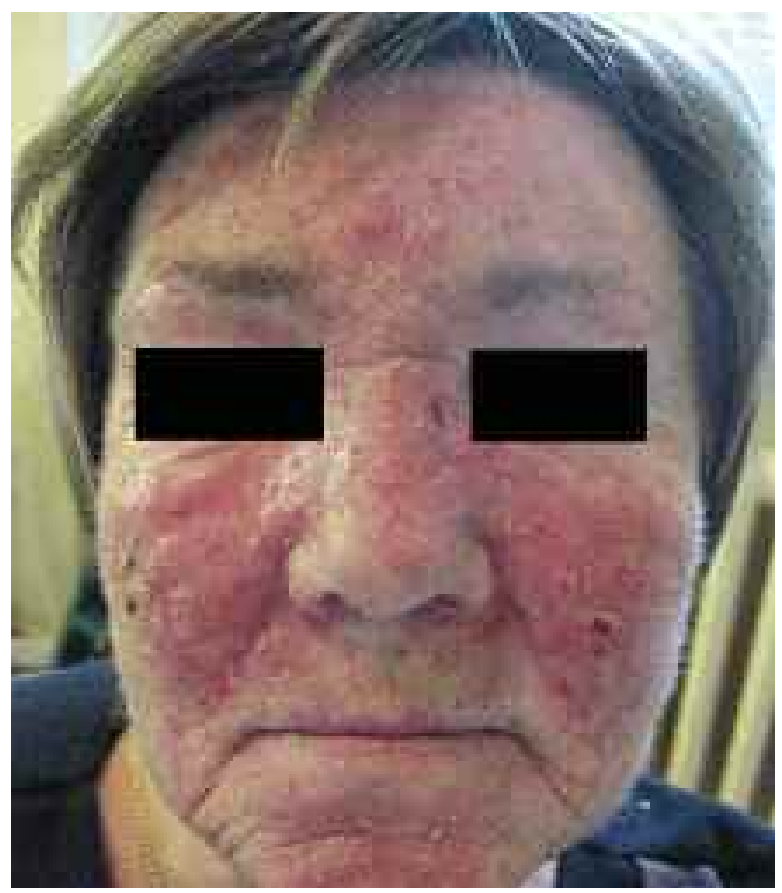

Figure 1. The papulopustular rash after 6 months of the treatment with cetuximab

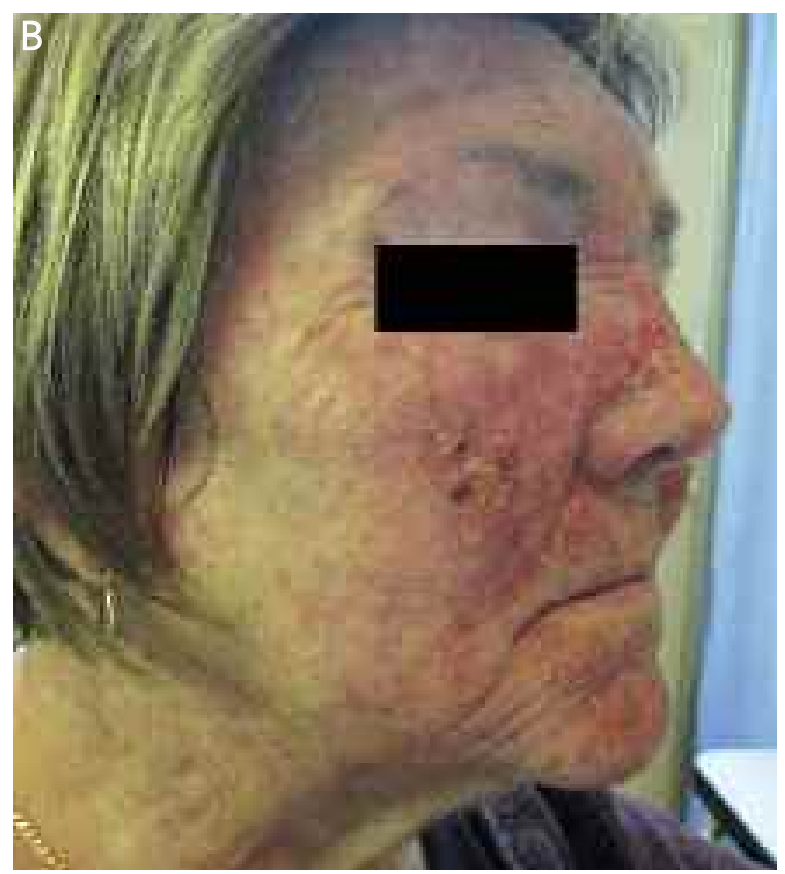

Figure 2 A-B. Multiple pustules and papules with severe erythema and impetiginisation 

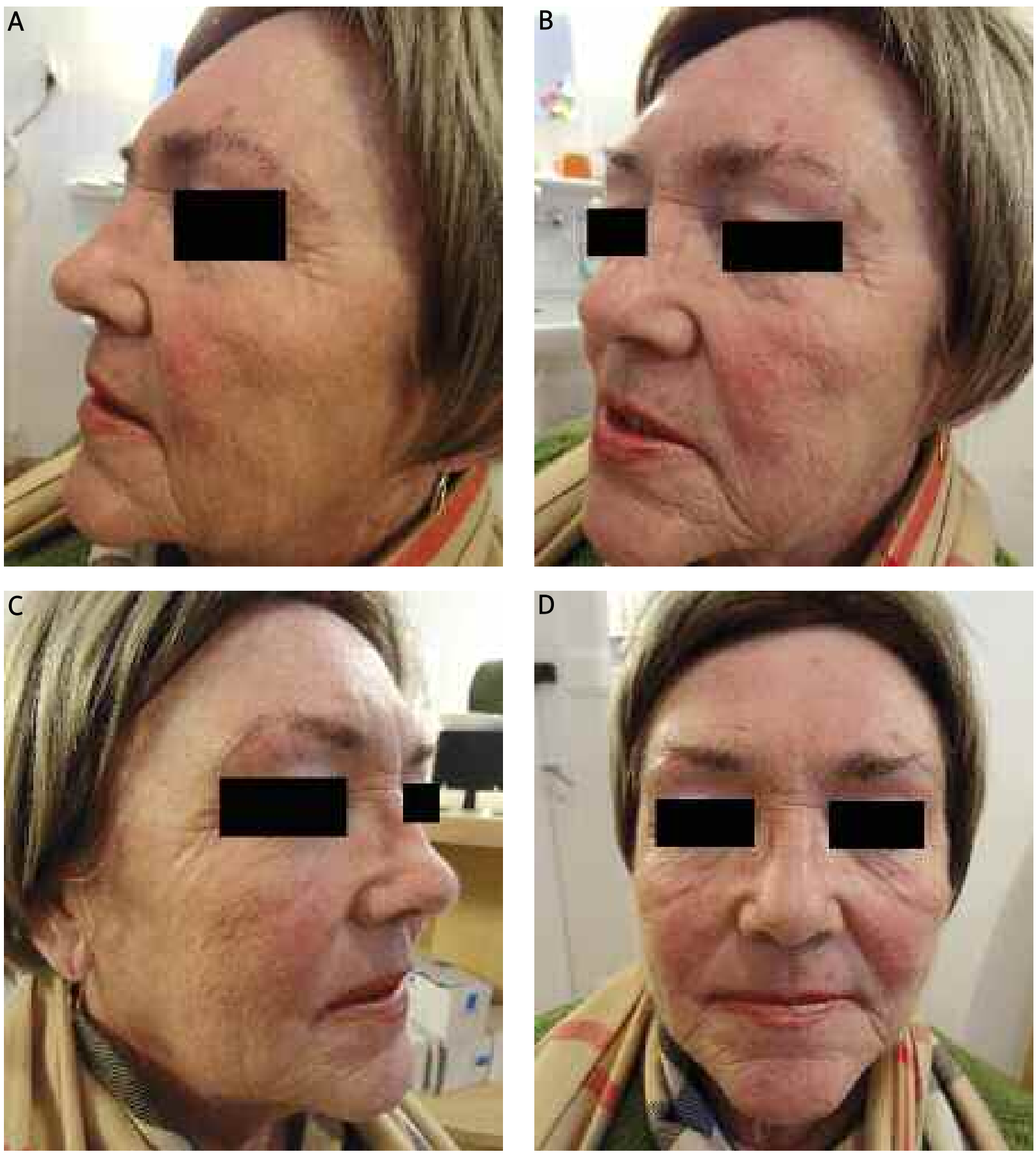

Figure 3 A-D. An improvement in the skin condition 4 weeks after the onset of dermatological treatment

of cetuximab. At that time facial skin lesions were mild and included mainly erythema and local irritation. Despite tendency to recurrence, the symptoms did not require discontinuing EGFR inhibitor therapy. However, the maintenance of antineoplastic treatment led over time to the exacerbation of the skin condition and development of typical papules and pustules.

Therapy consisting of oral doxycycline (100 mg twice daily) in association with topical tacrolimus ( $0.1 \%$ oint- ment) and fusidic acid (2\% cream) was given without cetuximab dose reduction. The papulopustular rash resolved within two weeks. The patient remains under control of the Dermatologic Clinic.

\section{Discussion}

Cetuximab is a chimeric monoclonal antibody which binds specifically to the EGFR. By competitively blocking 
ligand-dependent activation of EGFR, cetuximab inhibits phosphorylation and activation of receptor-associated kinases. Furthermore, it induces receptor internalization and consequent downregulation. Treatment with this drug reduces EGFR-dependent tumor cell proliferation, inhibits angiogenesis [8]. Studies have revealed that cetuximab mediates antibody-dependent cell-mediated cytotoxicity and has synergy with chemotherapy and radiation therapy $[5,8]$.

Cetuximab is indicated for the treatment of patients with EGFR-expressing, KRAS wild-type metastatic colorectal cancer ( $\mathrm{mCRC}$ ), in combination with chemotherapy, and as a single agent in patients who have failed oxaliplatin- and irinotecan-based therapy and who are intolerant to irinotecan. Cetuximab is also approved for the treatment of patients with squamous cell carcinoma of the head and neck in combination with platinum-based chemotherapy for the first-line treatment of recurrent and/or metastatic disease and in combination with radiation therapy for locally advanced disease. The study has shown that treatment with cetuximab as compared with best supportive care alone improved overall survival (median: 9.5 months vs. 4.8 months) and progressionfree survival (median: 3.7 months vs. 1.9 months) in patients with wild-type KRAS tumors [9].

\section{Dermatologic side effects of EGFR inhibitor therapy}

Despite the benefits of treatment, a number of dermatologic adverse events are experienced by patients undergoing therapy with cetuximab. They include papulopustular rash (acneiform rash), pruritus, xerosis, skin fissures, photosensitivity, telangiectasia, hyperpigmentation, paronychia, hair growth abnormalities, disorders of mucous membranes [10]. An incidence ranges from $80 \%$ to $95 \%$ of patients treated with cetuximab monotherapy. Trials of cetuximab combined with chemotherapy have revealed a similar frequency [11].

\section{Pathophysiology of skin reactions}

The inflammatory cell recruitment and the disruption of physiologic EGFR-mediated signaling processes in the epidermis and hair follicle are thought to be the mechanism by which EGFR inhibitors cause cutaneous toxicity [12].

The EGFR is expressed in the basal layer of the epidermis, hair follicles, sebaceous and eccrine glands. It plays a role in cell growth, differentiation and apoptosis, accelerates wound healing. Cetuximab binds to the EGFR on both normal and tumour cells. The proliferation of basal keratinocytes is decreased during EGFR inhibitor therapy. Investigations have shown a reduced expression of the proliferation marker Ki-67 and an elevated expression of the negative growth regulator p27 (cyclin-dependent kinase inhibitor) [12, 13]. Epidermal atrophy with the loss of the normal basketweave appearance of the stratum corneum is also associated with the apoptosis-induced effect of cetuximab. Furthermore, histological measures detect premature differentiation and dyskeratosis. The inflammatory chemoattractants like CXCLS and CCLs released by epithelial cells lead to the leukocyte activation and recruitment. Histopathologic analysis of the skin sample reveals folliculitis and destruction of follicles $[14,15]$.

\section{Clinical characteristics of papulopustular rash}

The most common cetuximab-induced dermatological reaction is papulopustular rash developing within two weeks of treatment onset. It begins with sensory disturbances, erythema and edema, followed by papulopustular eruption, crusting and quite often secondary infections. Lesions mainly localize in areas rich in sebaceous glands: the face, upper back and chest. Less frequently affected regions are the scalp, extremities, abdomen and buttocks. Although the rash reaches maximum intensity in 4-6 weeks after the onset of cetuximab therapy and decreases after 6-8 weeks, postinflammatory erythema or hyperpigmentation can be observed for months or years [16].

The EGFR inhibitor-induced eruption may resemble acne vulgaris with morphology and distribution. Some of terms, which signalize the acne-like appearance of the rash (such as acneiform rash), may suggest common etiology with acne. Therefore, these terms should not be used indeed but replaced with more adequate ones. The clinical appearance of the skin may also imitate other dermatologic diseases such as rosacea or seborrheic dermatitis.

The overall incidence of all-grade papulopustular rash ranges from $75 \%$ to $87 \%$ with $4-10 \%$ being high-grade [17]. An increased risk of developing this condition may be associated with the male gender and age [18].

Data show that there is a positive correlation between the development and severity of dermatological reactions and the clinical response to the EGFR inhibitor treatment $[19,20]$. Furthermore, the medial survival time is longer in patients with skin lesions. These findings suggest that cutaneous toxicity related to anti-EGFR therapy may be a clinical marker of effective treatment and predictor of survival.

Despite the benefits of EGFR-targeted agents, toxic effects of the skin may result in poor patients' compliance, dose modification, interruption or discontinuation of lifeprolonging antineoplastic therapy. Their noticeable impact on psychosocial well-being, quality of life, related costs and secondary infections are also noteworthy. In a survey of the oncologist, $32 \%$ of providers reported discontinuing therapy, $76 \%$ - holding it and $60 \%$ of oncology practitioners reduced the drug dose only due to rash [21].

Pharmacogenetics, an intensively developing branch of science based on genotype analysis, may select patients, who benefit from the treatment with the low- 
est risk of complications. Results of the studies confirm the association between genetic polymorphisms, the treatment adverse effects and the response of the tumour to monoclonal antibody targeted therapy. The analysed genes are related to EGFR signaling (EGF, EGFR, CCND1) or antibody-dependent cell-mediated cytotoxicity (FCGR2A, FCGR3A).

A number of $C A$ repeats in intron 1 of EGFR gene and point nucleotides substitution in the above genes have an impact on progression-free survival $[22,23]$. Another published study has shown the interaction between CA repeats polymorphism and grade of the skin toxicity induced by cetuximab [23].

\section{Grading scales of the dermatologic toxicity}

The most often used grading scale for evaluation of EGFR inhibitor-induced skin toxicity is the NCI-CTCAE (National Cancer Institute's Common Terminology Criteria for Adverse Events). It is based on 5 grades: grade 1 mild; grade 2 - moderate; grade 3 - severe or medically significant but not immediately life threatening; grade 4 - life-threatening consequences; grade 5 - death. However, this scale was not created for EGFR inhibitors and may cause incorrect assessment of dermatologic side effects. Underreporting the severity of some of them can lead to inadequate treatment. A Skin Toxicity Study Group of the Multinational Association of Supportive Care in Cancer designed a class-specific grading scale to improve reporting of EGFR inhibitor-associated dermatologic adverse events by detecting them with greater sensitivity, specificity and range. The proposed scale has the same terminology and grading principles as CTCAE version 4.0. However, it includes more specific descriptors of given conditions and patient-reported health-related quality of life factors [7].

The interpretation of the scale is individual. The studies concerning inter-observer agreement between dermatologists and oncologists in labeling and grading the cetuximab-induced dermatological toxicities has shown that both groups define by the same terms only $62 \%$ of cutaneous reactions. Also oncologists' grading of papulopustular eruption was lower [24]. Only multidisciplinary collaboration between oncologists and dermatologists may provide an optimal patient care.

\section{Prophylaxis and treatment recommendations}

Maintenance of the full-dose anti-EGFR therapy is a relevant goal of the approach to the cutaneous toxicity. There are only few randomized clinical trials investigating management of the EGFR inhibitor-associated skin side effects. Current recommendations are primarily based on published case reports, expert opinion and panel consensus [5, 16, 18].

Patient education prior to antineoplastic treatment is crucial for patients. General suggestions include limiting sun exposure and avoidance of activities and products that dry and irritate the skin (hot showers, alcohol-based perfumed products, over-the-counter acne medications). Sunscreens with high SPF containing zinc oxide or titanium dioxide are recommended regardless of the season. Moisturizing creams and emollients should be used daily to prevent and alleviate skin dryness [18, 25].

Based on the high frequency of the papulopustular rash within the first 2 weeks of therapy, MASCC Skin Toxicity Study Group recommends preventive measures. Prophylaxis is also important to avoid or minimize possible long-term sequelae after rash [16].

A randomized study has revealed that a preemptive regimen of moisturizer, sunscreen, hydrocortisone 1\% cream and doxycycline $100 \mathrm{mg}$ twice daily for the first 6 weeks reduces the risk of grade 2 and higher skin toxicity. Administration of doxycycline at the initiation of EGFR inhibitor therapy demonstrates better efficacy than reactive treatment after the appearance of the typical eruption [26]. One hundred $\mathrm{mg}$ daily of minocycline may be a useful agent in minimizing symptoms of the rash during the first month of cetuximab treatment. Oral antibiotics do not reduce the beneficial pharmacological effects of the anti-EGFR agents. Data have shown tazarotene to be ineffective. Topical retinoids are not recommended due to their drying effects [27].

The type, severity and location of the rash influence the decision of management in a given case. Individual response to treatment should also be considered. Therapeutic options include topical and systemic approaches.

Recommendations for the treatment of mild papulopustular rash include twice daily application of topical clindamycin 1-2\% combined with hydrocortisone $1 \%$ (aclometasone $0.05 \%$ or fluocinonide $0.05 \%$ suggested by MASCC Skin Toxicity Study Group) $[16,18]$. Skin reactions grade $\geq 2$ require an additional therapy with doxycycline (100 mg p.o. twice per day) or minocycline (100 mg p.o. twice per day) administered as long as rash is symptomatic [11]. Oral tetracyclines are recommended for their immunomodulating and anti-inflammatory effects. Doxycycline is preferred in patients with renal impairment. Minocycline is less photosensitizing but can cause loss of balance, hepatitis and a lupus-like syndrome. Guidelines for management of severe papulopustular rash are the same as those for moderate skin toxicity, with the addition of systemic steroids. Studies have suggested that oral retinoids in low-to-medium doses (20-40 mg for isotretinoin) decrease symptoms of the rash [18, 28]. Improved cellular differentiation and anti-inflammatory effects of the oral retinoids can play an important role in the treatment of eruption. However, side effects may reduce benefits over time [16].

Application of the calcineurin inhibitors is not recommended due to no significant benefit for the patient with EGFR inhibitor-induced rash [29]. Nevertheless, topical tacrolimus was administered in our patient due to 
facial location of the eruption, which is a contraindication for using topical steroids. An improvement in the skin condition was observed.

If a patient experiences pruritus, an oral antihistamine (hydroxyzine, cetirizine, loratadine) may be added [18].

In the case of severe skin reaction grade $\geq 3$, anti-EGFR therapy must be stopped. It may be resumed if the reaction has resolved to at least grade 2 . Dose reduction of EGFR inhibitor (cetuximab) is recommended at the second and third occurrence of grade 3 skin toxicity. Grade 4 reactions require discontinuing the therapy with monoclonal antibody [10].

In a recent study, a high-level laser therapy has been used in reducing the severity of facial papulopustular rash. This innovative method was effective with no side effects [30].

\section{References}

1. Klapper LN, Kirschbaum MH, Sela M, et al. Biochemical and clinical implications of the ErbB/HER signaling network of growth factor receptors. Adv Cancer Res 2000; 77: 25-79.

2. Schlessinger J. Cell signaling by receptor tyrosine kinases. Cell 2000; 103: 211-25.

3. Mendelsohn J, Baselga J. Status of epidermal growth factor receptor antagonists in the biology and treatment of cancer. J Clin Oncol 2003; 21: 2787-99.

4. Castillo L, Etienne-Grimaldi MC, Fischel JL, et al. Pharmacological background of EGFR targeting. Ann Oncol 2004; 15: 1007-12.

5. Keefe DMK, Bateman EH. Tumor control versus adverse events with targeted anticancer therapies. Nat Rev Clin Oncol 2011; 9: 98-109.

6. Pomerantz RG, Mirvish ED, Geskin LJ. Cutaneous reactions to epidermal growth factor receptor inhibitors. J Drugs Dermatol 2010; 9: 1229-34.

7. Lacouture ME, Maitland ML, Segaert S, et al. A proposed EGFR inhibitor dermatologic adverse event-specific grading scale from the MASCC skin toxicity study group. Support Care Cancer 2010; 18: 509-22.

8. Balagula Y, Garbe C, Myskowski P, et al. Clinical presentation and management of dermatological toxicities of epidermal growth factor receptor inhibitors. Int J Dermatol 2011; 50: 129-46.

9. Karapetis CS, Jonker DJ, O'Callaghan CJ, et al. K-ras mutations and benefit from cetuximab in advanced colorectal cancer. N Engl J Med 2008; 359: 1757-65.

10. Bianchini D, Jayanth A, Chua YJ, et al. Epidermal growth factor receptor inhibitor-related skin toxicity: mechanisms, treatment, and its potential role as a predictive marker. Clin Colorectal Cancer 2008; 7: 33-43.

11. Fakih M, Vincent M. Adverse events associated with antiEGFR therapies for the treatment of metastatic colorectal cancer. Curr Oncol 2010; 17: 518-30.

12. Lacouture ME. Mechanisms of cutaneous toxicities to EGFR inhibitors. Nat Rev Cancer 2006; 6: 803-12.

13. Segaert S, Van Cutsem E. Clinical signs, pathophysiology and management of skin toxicity during therapy with epidermal growth factor receptor inhibitors. Ann Oncol 2005; 16: 1425-33.

14. Nardone B, Nicholson K, Newman M, et al. Histopathologic and immunohistochemical characterization of rash to human epidermal growth factor receptor 1 (HER1) and HER1/2 inhibitors in cancer patients. Clin Cancer Res 2010; 16: 4452-60.

15. Mascia M, Mariani V, Girolami G, et al. Blockade of the EGF receptor induces a deranged chemokine expression in keratinocytes leading to enhanced skin inflammation. Am J Pathol 2003; 163: 303-12.

16. Wua PA, Balagulab Y, Lacouture ME. Prophylaxis and treatment of dermatologic adverse events from epidermal growth factor receptor inhibitors. Curr Opin Oncol 2011; 23: 343-51.

17. Su X, Lacouture ME, Jia Y, et al. Risk of high-grade skin rash in cancer patients treated with cetuximab-an antibody against epidermal growth factor receptor: systemic review and meta-analysis. Oncology 2009; 77: 124-33.

18. Lacouture ME, Anadkat MJ, Bensadoun RJ, et al. Clinical practice guidelines for the prevention and treatment of EGFR inhibitor-associated dermatologic toxicities. Support Care Cancer 2011; 19: 1079-5.

19. Lenz HJ, Van Cutsem E, Khambata-Ford S, et al. Multicenter phase II and translational study of cetuximab in metastatic colorectal carcinoma refractory to irinotecan, oxaliplatin and fluropyrimidines. J Clin Oncol 2006; 24: 4814-921.

20. Racca P, Fanchini L, Caliendo V, et al. Efficacy and skin toxicity management with cetuximab in metastatic colorectal cancer: outcomes from an oncologic/dermatologic cooperation. Clin Colorectal Cancer 2008; 7: 48-54.

21. Boone SL, Rademaker A, Liu D, et al. Impact and management of skin toxicity associated with anti-epidermal growth factor receptor therapy: survey results. Oncology 2007; 72: 152-9.

22. Pander J, Gelderblom H, Antonini NF, et al. Correlation of FCGR3A and EGFR germline polymorphisms with the efficacy of cetuximab in KRAS wild-type metastatic colorectal cancer. Eur J Cancer 2010; 46: 1829-34.

23. Dahan L, Norguet E, Etienne-Grimaldi MC, et al. Pharmacogenetic profiling and cetuximab outcome in patients with advanced colorectal cancer. BMC Cancer 2011; 11: 496.

24. Duffour J, Thézenas S, Dereure O, et al. Inter-observer agreement between dermatologists and oncologists in assessing dermatological toxicities in patients with metastatic colorectal cancer treated by cetuximab-based chemotherapies: a pilot comparative study. Eur J Cancer 2010; 46: 3169-74.

25. Waris W, Naik S, Idrees I, et al Severe cutaneous reaction to cetuximab with possible association with the use of overthe-counter skin care products in a patient with oropharyngeal cancer. Cutan Ocul Toxicol 2009; 28: 41-8.

26. Lacouture ME, Mitchell EP, Piperdi B, et al. Skin toxicity evaluation protocol with panitumumab (STEPP), a phase II, openlabel, randomized trial evaluating the impact of a pre-emptive skin treatment regimen on skin toxicities and quality of life in patients with metastatic colorectal cancer. J Clin Oncol 2010; 28: 1351-7.

27. Scope A, Agero AL, Dusza SW, et al. Randomized double-blind trial of prophylactic oral minocycline and topical tazarotene for cetuximab-associated acne-like eruption. J Clin Oncol 2007; 25: 5390-6.

28. Pomerantz RG, Chirinos RE, Falo LD, et al. Acitretin for treatment of EGFR inhibitor-induced cutaneous toxic effects. Arch Dermatol 2008; 144: 949-50.

29. Scope A, Lieb J, Dusza S, et al. A prospective randomized trial of topical pimecrolimus for cetuximab-associated acne-like eruption. J Am Acad Dermatol 2009; 61: 614-20.

30. Gobbo M, Ottaviani G, Mustacchi G, et al. Acneiform rash due to epidermal growth factor receptor inhibitors: high-level laser therapy as an innovative approach. Lasers Med Sci 2011 [Epub ahead of print]. 